\title{
Study on the Surgical Scheduling Problem Considering the Uncertainty of Operation Time
}

\author{
Tang Rui ${ }^{1, a}$, Lv Jie ${ }^{2, b^{*}}$ \\ ${ }^{1}$ School of Economics and Management, The Chinese University of Jiangsu Science and Technology, Dantu, \\ Zhenjiang, Jiangsu, China \\ ${ }^{2}$ School of Economics and Management, The Chinese University of Jiangsu Science and Technology, Dantu, \\ Zhenjiang, Jiangsu, China \\ a695744330@qq.com \\ b*458224479@qq.com
}

\section{ABSTRACT}

Surgery is the top priority in medical work. According to incomplete statistics, surgery will involve $70 \%$ of the hospital's entire resources. Although China's surgical construction has achieved certain successes, due to differences in the initial time and end time of surgery Certainly, the patient's arrival time is uncertain, and the operating room, hospital beds, medical equipment, medical workers and other resources are constrained. There are still the following problems: relatively scarce surgical resources, low utilization of surgical beds, increasing conflicts between doctors and patients, and surgeries The outage rate is high. In order to better solve the problems in surgical work, ease the contradiction between surgical supply and demand, and use surgical resources reasonably and efficiently, we need to study the problem of surgical scheduling.

Keywords: Surgery, uncertain time, surgical scheduling, surgical resources

\section{考虑手术时间不确定性的手术调度问题研究}

唐锐 $1, \mathrm{a}$ 吕洁 $2, \mathrm{~b}^{*}$

\footnotetext{
1 江苏科技大学经济管理学院, 丹徒, 镇江, 江苏, 中国

2 江苏科技大学经济管理学院, 丹徒, 镇江, 江苏, 中国

a695744330@qq.com

b*458224479@qq.com
}

摘要

手术是医疗工作中的重中之重，据不完全统计，手术会涉及到医院整个资源的百分之七十,虽说我国的手术建 设取得一定的成就，但由于手术初始时间和结束时间的不确定、病人到达的时间不确定，手术室、病床、医疗 设备、医护工作者等资源的约束性，仍存在以下问题：手术资源相对贵乏，手术病床的利用率低，医患矛盾不 断增加, 手术的停台率高。为了更好的解决手术工作中的问题, 缓解手术供需矛盾, 合理高效的利用手术资源, 所以我们需要研究手术调度问题。

关键词：手术，时间不确定，手术调度，手术资源

\section{1.前言}

手术是医疗工作中的重中之重，据不完全统计， 手术会涉及到医院整个资源的百分之七十, 虽说我国 的手术建设取得一定的成就，但由于手术初始时间和 结束时间的不确定、病人到达的时间不确定, 手术室、
病床、医疗设备、医护工作者等资源的约束性，仍存 在以下问题: 手术资源相对贵乏，手术病床的利用率 低，医患矛盾不断增加，手术的停台率高。为了更好 的解决手术工作中的问题，缓解手术供需矛盾，合理 高效的利用手术资源，所以我们需要研究手术调度问 题。 
目前学者根据不同影响因素进行了大量的手术 调度问题研究，(1) 时间偏好: 李云等认为医生首先 是一个利他主义者, 医疗服务是一种利他主义行为 ${ }^{[1]}$; 偏好是行为的理由, 医生在进行手术选择时, 除了利 他之外，还遵循其他偏好，而时间偏好则是医生众多 偏好中的一种 ${ }^{[2]}$; 医生的时间偏好是指医生对被安排 手术任务的时间的偏好, 包括医生的时间可用性以及 医生对不同时间区间的偏好 [3]。（2）手术时间：由 于手术的难易程度不同，导致手术时间具有不确定性， 王昱等基于手术服务时间不确定性，以院方权益最大 为目标, 建立了手术调度问题的确定型模型, 进一步 考虑了管理者风险偏好对患者服务时间不确定集的 影响, 提出了区间型手术调度问题的两阶段鲁棒优化 方法 ${ }^{[4]}$; 王珊珊等考虑到手术时间的不确地性, 提出 手术室加班时间的机会约束, 以一定概率保证病人的 手术时间不超过手术室的开放时间, 建立随机优化机 会约束手术计划调度模型, 确定手术室的开放和分配 决策 ${ }^{[5]}$; 王恺等基于病人手术过程中服务时间的不确 定性, 建立了可重入手术调度问题的数学模型, 以期 降低病人术后恢复的平均完成时间 ${ }^{[6]}$; 杨枫等针对应 急救援情境下手术调度中存在不确定性因素导致无 法获得精确的手术时间和结束期的问题, 设计了一种 灰色调度模型和求解该问题的混合灰色布谷鸟算法 ${ }^{[7]}$; Liu 等通过拟合分布预测手术时间, 以提高手术室 的利用率, 降低手术成本及提高医生的满意度为优化 目标进行手术安排 ${ }^{[8]}$; Wang 等 (2021) 研究了手术时 间不确定和病床短缺情况下的手术调度 ${ }^{[9]}$; (3) 医护 人员资源: 手术人员具有专业性, 同一个时间段, 一 个医护人员只能做一台手术, 因此, 医护人员是手术 调度中不可忽略的因素。贺天勇等在护士资源不确定 的情况下, 采用基于 pareto 解集的蚁群算法, 研究手 术排程重调度问题 ${ }^{[10]}$; 刘洪伟等考虑到不同科室病人 的医学特征带来的术间和医生的约束, 建立了手术排 程和医生指派问题的数学模型, 确定每个术间的手术 序列并为病人指派符合要求的医生 ${ }^{[11]}$; 罗永等以手术 室的均衡利用为目标, 建立考虑同一个医生的手术安 排在同一个手术室和同一个医生的手术可以安排在 不同手术室两种情况下的混合整数规划模型 ${ }^{[12]}$; 成舒 凡等为了解决突发事件下应急手术调度问题, 将应急 手术调度问题看作混合流水车间调度问题, 并考虑了 医护人员长时间工作而带来的疲劳效应和截断学习 效应, 构建了术前、术中、术后三阶段手术调度模型 ${ }^{[13]}$; Silva 等研究基于医护工作人员特殊技能和工作 时间进行手术安排 ${ }^{[14]}$ 。

本文基于上述文献的研究，考虑到了手术时间的 不确定的手术调度问题, 建立以安排患者手术, 使得 手术计划不能完成的风险最小和使患者在住院等待 手术期间病情感染或恶化的风险最小为目标的多目 标非线性规划模型。通过多目标最优化算法的三个评 价指标, 发现解的分布均匀性较好, 找到的帕累托前 沿与真正帕累托前沿近似贴合, 证明了粒子群算法在 解决手术排程问题上具有较为良好的性能, 求解出手 术调度的最优解。

\section{2. 问题假设与模型建立}

\section{1. 问题描述}

因为患者的身体状况、医生的手术技能及手术过 程中的多种不确定性因素有关，导致手术时间具有不 确定性。本文用平均值和方差来表示患者预期的手术 时长 。由于用单一时间估计手术时长存在较大的误 差, 大大降低了排程的可行性; 因此本文采用三点时 间估计法, 分别估计手术时长的最乐观时间、最可能 时间以及最保守时间, 然后利用这三个时间估算手术 时长的平均值和方差。其中最乐观时间, 以 ta 表示, 指在顺利情况下完成任务所需的最短时间: 最保守时 间, 以 tb 表示, 指在最不利情况下完成任务所需的最 长时间; 最可能时间, 以 $\mathrm{tm}$ 表示, 指在一般情况下 完成任务所需的最可能时间。

已知某科室有 $\mathrm{N}$ 位患者等待手术, $\mathrm{F}$ 位主治医生, $\mathrm{J}$ 个可用手术室; 用均值 $\mathrm{dt}$ 和标准差 ${ }^{i}$ 来表示患者 $\mathrm{i}$ 的手术时长。所有手术室的规定日开放时间相同, 均 为 $\mathrm{c}$ 小时。患者的主治医生已选定, 用 0-1 变量 pit 表 示, pit $=1$ 表示患者 $\mathrm{i}$ 的主治医生为 1 , 否则 $\mathrm{pit}=0$; 医 生的日最大工作量不超过 $\mathrm{s}$ 小时。依照患者病情, 由 主治医生给定患者的病情易感染系数 wi, wi 越大, 患者的感染风险越大; 医院通常采用一周 5 个工作日 为周期，对患者进行手术排程。

目标如下:

目标一: 如何安排患者手术，使得手术计划不能 完成的风险最小。

目标二: 如何安排患者手术，使得患者在住院等 待手术期间病情感染或恶化的风险最小，即尽量将有 感染或恶化风险的患者安排在前面手术。

\section{2. 模型建立}

针对目标一: 设手术室 $\mathrm{j}$ 第 $\mathrm{k}$ 天无法完成手术计 划的风险为 rik，则目标函数可以表示为:

$$
\min R=\sum_{j=1} \sum_{k=1} r_{j k}
$$

为计算 rik, 假设手术室一天的手术时间 xjk 服从 正态分布，定义随机变量：

$$
\left\lfloor\left(X_{j k}, \Gamma_{j k}\right), X_{j k}, \Gamma_{j k}\right\rfloor
$$

即为手术室 $\mathrm{j}$ 第 $\mathrm{k}$ 天使用时间的平均值和标准差, 将它们表示为:

$$
X_{j k}=\sum_{i=1}^{N} d_{i} * x_{j k}, \Gamma_{j k}=\left(\sum_{i=1}^{N} \sigma_{1}^{2} * x_{j k}\right)^{1 / 2}
$$

则手术计划可以完成的概率为: 
$P\left(X_{j k}<c\right)=P\left(\frac{X_{j k}-X_{j k}}{\Gamma_{j k}}<\frac{c-X_{j k}}{\Gamma_{j k}}\right)=\phi\left(\frac{c-X_{j k}}{\Gamma_{j k}}\right)$

则:

$$
r_{j k}=1-\phi\left(\frac{c-X_{j k}}{\Gamma_{j k}}\right)
$$

相应的正态分布图如下:

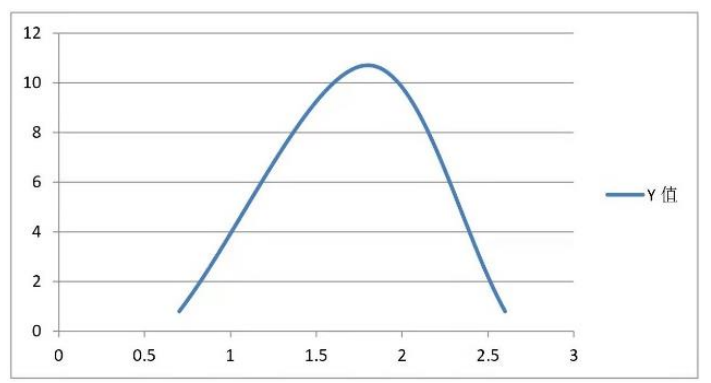

图 1 手术室日使用时间分布图

由图发现，通过减小手术时间的标准差，减小手 术任务未完成的风险。假设有两个手术室-日期, 每个 手术室-日期均被安排两次手术, 其中两位患者的手 术时间为 $(d, c)=(100,10)$, 另两位患者的手术时 间为 $(d, o)=(100,50)$, 比较以下两种情况的标准 差。如上图所示, 在第一种情况下, 手术室日工作时 间标准差均为:

$$
\sqrt{\left(50^{2}+10^{2}\right)=51}
$$

则标准差总和为 102 , 第二种情况下, 手术室日 工作时间标准差为:

$$
\sqrt{\left(50^{2}+50^{2}\right)+\left(10^{2}+10^{2}\right)=84.9}
$$

有效的减小了手术室日工作时间标准差, 降低了 手术计划无法完成的风险。

针对目标二: 尽量将病情有感染或恶化危险的手 术安排在前面, 即患者的病情感染或恶化系数越高, 他的手术日期应该越早。患者 $\mathrm{i}$ 的手术日期可以表示 为:

$$
T_{\mathrm{i}}=\sum_{j=1}^{J} \sum_{k=1}^{k} k_{x i j k}
$$

对于任何两个不同的手术任务 i1 和 i2, 满足 wi1 $>$ wi2, 若我们能够得到决策结果:

$$
T_{i 2} \leq T_{i 2}
$$

则认为达到了第二个目标。因此, 我们可以用下 式来表示第二个目标:

$$
\min W=\sum_{i=1}^{N} \sum_{j=1}^{J} \sum_{k=1}^{k} w_{i} * k_{x i j k}
$$

简化为:

$$
\min W=\sum_{i=1}^{N} w_{i} * T_{i}
$$

\section{3. 结果与分析}

本文问题模型为非线性多目标, 采用一般的数学 规划方法难以求解，而粒子群算法本身具有快速收敛 性和高维多目标优化能力, 参数少易于实现, 因此我 们采用群体智能中粒子群算法来对问题进行求解。

算法的运行是否成功, 探索能力和开发能力的平 衡是非常关键的。对于粒子群算法来说, 这两种能力 的平衡就是靠惯性权重来实现。较大的惯性权重使粒 子在自己原来的方向上具有更大的速度, 从而在原方 向上飞行更远, 具有更好的探索能力; 较小的惯性权 重使粒子继承了较少的原方向的速度, 从而飞行较近, 具有更好的开发能力。学习因子使得粒子具有自我总 结和向群体中优秀个体学习的能力, 从而向群体内或 领域内最优点靠近, PSO 搜索过程分为三阶段：以 PSO 最大的迭代次数为衡量指标:

（1） 0-10\%: 自由搜索阶段，粒子基本可以自由 的对解空间进行探索;

（2） $10 \%-40 \%$ : 调整阶段，粒子开始加强在某些 局部区域的开发工作;

（3） 40\%-100\%:收敛阶段，粒子被几个局部最 优解所吸引, 种群趋向收玫。

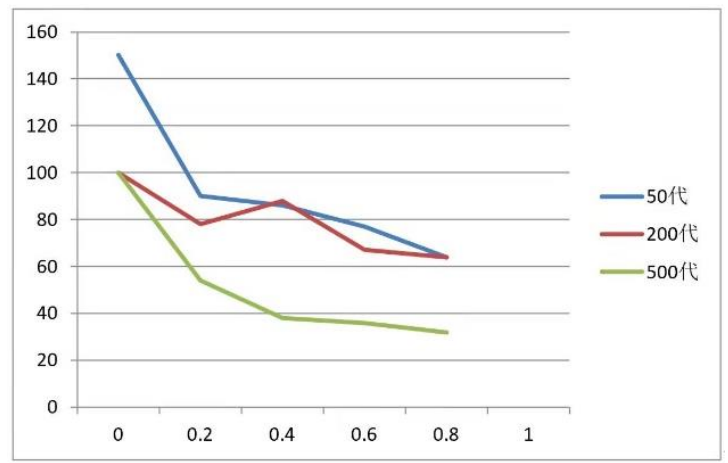

图 2 算法收敛性能图

这里将近似最好的解在目标函数空间的映像称 为精确 Pareto 前沿, 即在不同参数取值和种群规模下, 多次长期迭代求得的解在目标函数空间的映像, 如图 中紫色线标注。将一次运行得到的非支配解集在目标 函数空间的映像称为找到的帕累托前沿, 如三角号线 标记。由多目标最优化算法的三个评价指标, 从算法 中找到最优解个数为 7 个, 达优率为 $33.3 \%$, 如表 3.1 所示, 除端点数例 $(0.0036,100)$ 与 $(0.008,98)$ 外, 解的分布均匀性较好, 找到的帕累托前沿与真正帕累 
托前沿近似贴合, 证明了粒子群算法在解决手术排程 问题上具有较为良好的性能。

\section{表 1 算法最优解}

编号目标1 目标2 最优解

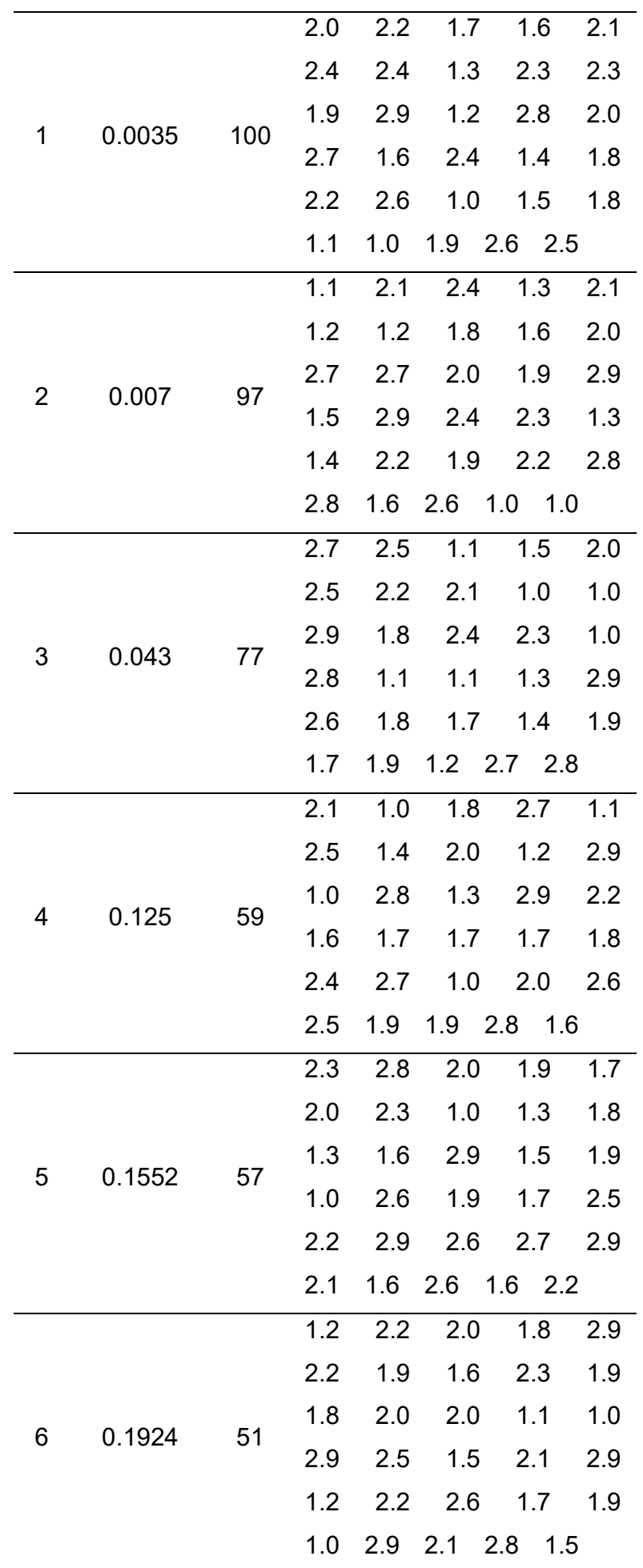

\begin{tabular}{|c|c|c|c|c|c|c|c|}
\hline \multirow{6}{*}{7} & \multirow{6}{*}{0.2908} & \multirow{6}{*}{37} & 2.3 & 2.7 & 2.9 & 2.9 & 1.6 \\
\hline & & & 1.9 & 1.8 & 1.5 & 2.1 & 1.7 \\
\hline & & & 1.5 & 2.0 & 1.9 & 1.9 & 1.1 \\
\hline & & & 1.7 & 2.6 & 2.2 & 1.0 & 2.6 \\
\hline & & & 2.0 & 2.7 & 2.5 & 2.8 & 2.8 \\
\hline & & & 1.3 & 2.1 & 1.6 & 1.8 & \\
\hline
\end{tabular}

以编号为 1 的最优解为例, 说明解的物理含义: 解中元素的位置表示患者编号; 解中每个元素的个位 表示患者被分配的手术室编号; 十分位表示患者被指 派的手术日期，其中 0-4，5-9 分别表示周一到周五 5 个工作日。如编号为 1 的最优解: 其中第一个元素 2.0 表示患者 1 被安排到手术室 2 手术，手术时间为周 一; 第二个元素 2.2 表示患者 2 被安排到手术室 2 手 术, 手术时间为周三, 依次类推。在多个最优解当中, 医院管理者根据自身偏好和发展战略选择排程方案。

若管理者期望手术计划未完成的风险尽量小，而 患者等待手术期间感染因子控制在一定范围内即可, 则可选择最优解 1, 2; 若管理者期望兼顾两个目标, 将计划未完成风险和患者等待手术期间感染风险均 控制在一定标准内, 则可选择最优解 3、4、5; 若医 院管理者力争达到患者入院后零风险的治疗目标, 医 护人员乐于适当加班完成手术任务, 创出医院口碑和 品牌, 则可选择最优解 6、7。

\section{4. 结束语}

本文通过考虑手术时间不确定的手术调度问题, 建立以最小化安排患者手术，使手术计划不能完成的 风险和最小化使患者在住院等待手术期间病情感染 或恶化的风险为多目标的非线性规划模型。通过采用 粒子群优化算法求解出手术调度的最优解, 为决策者 制定最优的手术排程计划提供了参考依据, 从而提高 手术室的利用率, 为医院增加收益。

\section{REFERENCES}

[1] Li Yun. Ge Jian: Doctors must be altruists[N]. Guangdong Science and Technology News,2008-0624(A03).

[2] Song Chaoying, Zhu Jianming, Yan Aili. Behavioral Economics Explanation of Inconsistent Time Preference[J]. Economic Issues, 2011(01):13-19.

[3] Penn M L, Potts C N , Harper P R . Multiple criteria mixed-integer programming for incorporating multiple factors into the development of master operating theatre timetables[J]. European Journal of Operational Research, 2017, 262(1):194-206.

[4] Wang Yu,Tang Jiafu.Research on two-stage robust optimization method for hospital operation scheduling problem[J].Journal of Systems Engineering,2016,31(04):431-440.

[5] Wang Shanshan,Li Jinlin,Peng Chun,Ran Lun.Surgery 
planning stochastic model and algorithm based on chance constraints[J].System Engineering Theory and Practice,2019,39(07):1721-1731.

[6] Wang Kai,Chen Xia,Chen Lijun.Scheduling of reentrant surgery under service time changes[J].Operations Research and Management,2020,29(11):144-151.

[7] Yang Feng,Ye Chunming,Wang Dingyu.Solving Uncertain Operation Scheduling Model Based on Hybrid Cuckoo Algorithm[J].Application Research of Computers,2020,37(03):768-773.

[8] Liu H, Zhang T, Shuai L, et al. Operating room scheduling and surgeon assignment problem under surgery durations uncertainty[J]. Technology \& Health Care, 2017, 26(2):297-304.

[9] Wang J J, Dai Z, Chang J, et al. Surgical scheduling by Fuzzy model considering inpatient beds shortage under uncertain surgery duration[J]. Annals of Operations Research, 2021.

[10] He Tianyong,Xiang Wei.Surgery scheduling rescheduling under uncertainty of nurse resources[J].Technology and Management,2014,16(03):48-52.

[11] Liu Hongwei,Zhang Tianyi,Xu Dan.Study on multidepartment surgery schedule and doctor assignment[J].Industrial Engineering and Management,2016,21(06):10-16.

[12] Luo Yong,Luo Li,Zhou Ying, You Yang.Comparative study of surgical scheduling under mixed integer programming[J].Industrial Engineering and Management,2016,21(02):146-149+162.

[13] Cheng Shufan, Ye Chunming.Research on emergency surgery scheduling embedded with fatigue effect and learning effect[J].Computer Engineering and Applications, 2021, 1(10):06-22.

[14] Silva, Thiago AO, et al. "Surgical scheduling with simultaneous employment of specialised human resources." European Journal of Operational Research, 2015, 3 (245): 719-730. 